\title{
Wohin geht der Weg im OP?
}

\section{Gedanken einer leitenden OP-Fachkraft zu Beginn des neuen Jahrhunderts}

\author{
A. Karasek
}

\section{Zusammenfassung}

Ein Umdenken zur Veränderung unserer OP-Welt für die Zukunft ist unerlässlich. Die Forderung zur interdisziplinären Zusammenarbeit im OP ist sofort anzustreben. Unsere Kunden haben den Anspruch nach dem heutigen Stand der Ausbildung, des Materials und der Technik, die bestmögliche Versorgung zu erfahren. Verschließen wir uns nicht allen Neuheiten. Stellen wir uns den Anforderungen für die Zukunft und erhalten uns einen hoch sensiblen immer zu respektierenden Arbeitsplatz.

\section{Einleitung}

Viele Fragen stehen für die Zukunft offen. Die Berufspolitik im Gesundheitssystem braucht Innovation, es kann nicht sein, dass die Ausbildung bis zu einer OP-Fachkraft 7 Jahre dauert.

TOA (Technischer Operations Assistent) 3 Jahre. Die Verdienstmöglichkeiten gleichgestellt.!?

Die Interdisziplinäre Zusammenarbeit im $\mathrm{OP}$, Anästhesie - OP, Ambulanz - Intensiv - OP.

Die Integration neuer Technologien, eine sinnvolle Organisation/Koordination unter Berücksichtigung der laufenden Kosten von Personal, Material und technischer Ausstattung.

OP-JOURNAL 2000; 16: 194-195

(c) Georg Thieme Verlag Stuttgart · New York

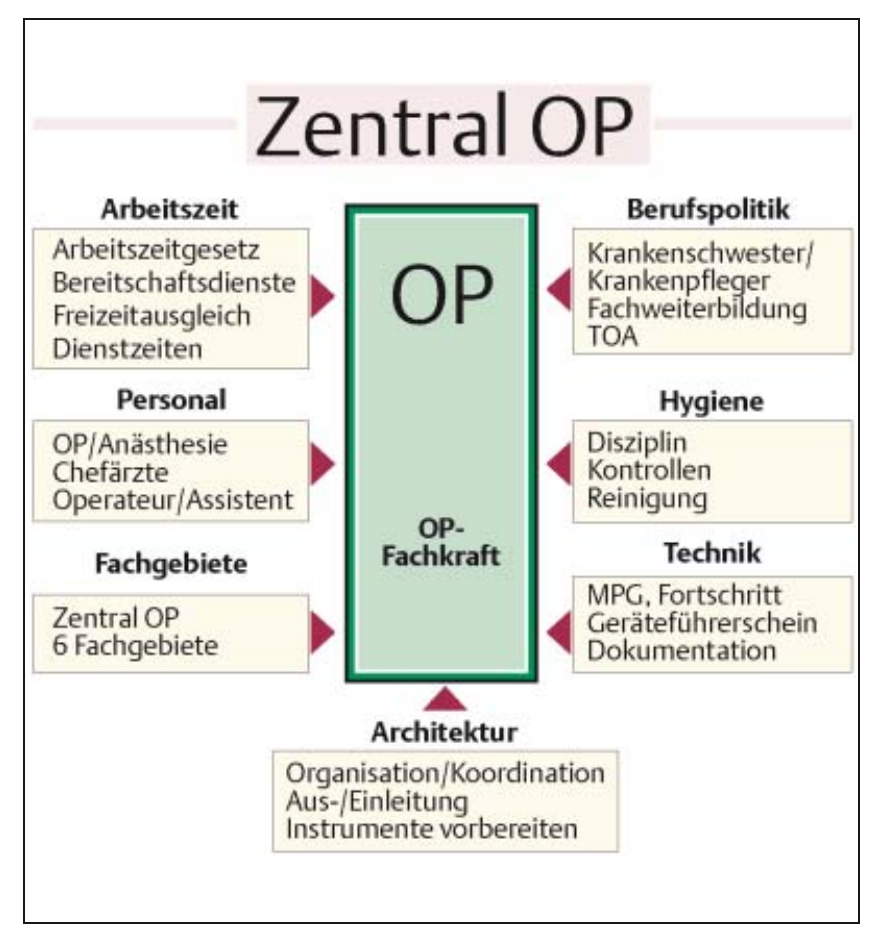

Abb.1 Qualifikation OP-Fachkraft.

\section{OP - Management Prozesssteuerung, Qualitätssicherung}

Eine optimale Auslastung unserer OP's im täglichen Betrieb fordern organisatorische Höchstleistung der verantwortlichen OP-Programm-Planer, OP-Koordinatoren und Leitungen. Nur eine partnerschaftliche Bereitschaft unter allen Beteiligten lassen eine Verbesserung zu.

EDV gesteuerte Dokumentationssysteme tragen erheblich zu einer genauen und korrekten Erfassung der erbrachten Leistungen während des OP-Aufenthaltes unserer Kunden bei.

Die Zuordnung aller Kosten, Personal, Material und Gerätschaften ermöglichen eine fallbezogene Abrechnung. Eine automatisierende Lösung ist hier anzustreben.

\section{Integration neuer Technologien}

Neue technische Entwicklungen zu einer schonenden Behandlung unserer Patienten durch z. B. minimal-invasive Möglichkeiten, endoskopische, sowie navigierte Operationen.

Bessere anatomische funktionelle Ergebnisse, sowie eine schnellere Genesung der Patienten müssen zum Ziel erklärt werden. Roboter und Computer werden uns in Zukunft im OP noch besser beschäftigen.

Weiterentwicklung und Verbesserung der AO-Synthesen fordern eine ständige Fort- und Weiterbildung des OP-Personals. Stetige neue und aktuelle Informationen müssen, z. B. durch das OP-Journal, die Basis aller OP-Mitarbeiter erreichen. 


\section{Herausforderung zur Interdisziplinären Zusammenarbeit}

Der Patient geht uns alle an.

Hochqualifizierte Mitarbeiter aller Berufsgruppen im OP, müssen verstärkt interdisziplinär zusammenarbeiten. Fachübergreifende Tätigkeiten sind in zeitlichen Reserven gemeinsam möglich.

Die Wechselzeiten zwischen den Operationen zu verkürzen sowie den erforderlichen Personalaufwand pro Operation zu reduzieren.

OP-Personal-Fachbereiche zu einer Operation, mögliche Einsparung während Schnitt-Nahtzeit.

\begin{tabular}{lll} 
Vorbereitung & Schnitt-Nahtzeit & Nachbereitung \\
\hline Instrumenteur & Instrumenteur & Instrumenteur \\
Springer & Springer/AN-Pflege & Springer \\
AN-Pflege & & AN-Pflege \\
Operateur/Assistent & Operateur/Assistent & Operateur/Assistent \\
Anästhesist & Anästhesist & Anästhesist \\
& & Reinigung
\end{tabular}

Wechselzeiten verkürzen, mögliche Personaleinsparung bei fachlicher Spezialisierung und Standardisierung.

\begin{tabular}{lll}
\hline Vorbereitung/Nachbereitung & Schnitt-Nahtzeit & Nachbereitung \\
\hline $\begin{array}{l}\text { Zentrale Narkose } \\
\text { Ein- Ausleitung }\end{array}$ & Instrumenteur & Instrumentenreinigung \\
Ausleitung & Operateur/Assistent & \\
& Anästhesie & $\begin{array}{l}\text { Springer } \\
\text { Reinigung }\end{array}$ \\
$\begin{array}{l}\text { Zentrale Instrumentenvor- } \\
\text { bereitung zur Operation }\end{array}$ & Instrumenteur/Springer & \\
& (Standardtisch) &
\end{tabular}

Zu diesen Einsparungsmöglichkeiten müssen auch die baulichen Voraussetzungen vorhanden sein.
A. Karasek

OP-Leitung und Koordinator

Klinikum Kempten-Oberallgäu GmbH Robert-Weixler-Str. 50

87439 Kempten (Allgäu) 